\title{
Top-down and Bottom-up Evaluation Procedurally Integrated
}

\author{
DA VID S. WARREN \\ Stony Brook University, Stony Brook, NY, 11794-4400 USA \\ (e-mail: warren@cs.stonybrook.edu) \\ XSB Inc., Setauket, NY, 11733 USA \\ (e-mail: warren@xsb.com)
}

submitted 30 April 2018; accepted 11 May 2018

\begin{abstract}
This paper describes how the Logic Programming System XSB combines top-down and bottomup computation through the mechanisms of variant tabling and subsumptive tabling with abstraction, respectively.

It is well known that top-down evaluation of logical rules in Prolog has a procedural interpretation as recursive procedure invocation (Kowalski 1986). Tabling adds the intuition of short-circuiting redundant computations (Warren 1992). This paper shows how to introduce into tabled logic program evaluation a bottom-up component, whose procedural intuition is the initialization of a data structure, in which a relation is initially computed and filled, on first demand, and then used throughout the remainder of a larger computation for efficient lookup. This allows many Prolog programs to be expressed fully declaratively, programs which formerly required procedural features, such as assert, to be made efficient.
\end{abstract}

KEYWORDS: top-down, bottom-up, logic programming, tabling, Prolog, procedural interpretation

\section{Introduction}

Top-down and bottom-up algorithms have long been seen as alternative, even competitive, ways to evaluate Datalog (and Prolog) programs (Lloyd 1993; Bry 1990), with proponents of each algorithm arguing for its particular advantages (Ullman 1989; Toman 1995; Tekle and Liu 2011). Systems implement either top-down evaluation, e.g., XSB (by default) (Swift and Warren 2012) and other Prolog systems that include tabling, such as YAP (Santos Costa et al. 2012), Ciao Prolog (Hermenegildo et al. 2012), BProlog (Zhou 2012) and as a library in SWI Prolog (Weilemaker et al. 2017), or bottom-up evaluation, e.g., LDL++ (Arni et al. 2003), Coral (Ramakrishnan et al. 1994) and LogicBlox (Aref et al. 2015), among many others. In this paper we consider the properties and advantages of each strategy and propose that they be integrated in a single system, with each having a distinct procedural interpretation. Then each can be used when its evaluation strategy is most appropriate and efficient. This paper describes how they are integrated in the XSB tabled logic programming system. 


\section{Example of Need for Bottom-Up Evaluation}

\section{Example 3}

Consider a simple system with a corpus of sentences, which allows a user to input a sentence and find all the sentences in the corpus that share a word with the input sentence. The system uses a relation of strings, called corpus (CorSent), which contains the sentences of the corpus, and defines a predicate share (+InSent,-CorSent, -Word), that, given an input sentence, returns each corpus sentence that shares a word with it, and the shared word. It can be defined using a helper tokenizing predicate, scan/2, as follows:

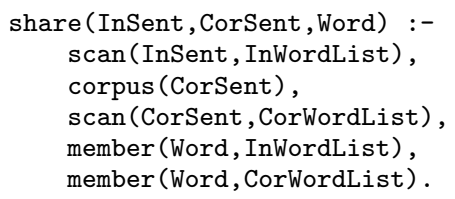

Top-down evaluation by Prolog proceeds as follows: Given a string as input, scan/2 tokenizes it, providing the list of words it contains. Then for each sentence in the corpus, we tokenize it, and return the corpus sentence for each word in the input sentence token list that is also in the corpus sentence token list.

This is a reasonable approach for a single query, but if we want to answer multiple similar queries to the same corpus, this will re-tokenize every string in the corpus for each query, which would be extremely redundant and inefficient: Answering a single query for one input sentence essentially requires almost all the work (i.e., tokenizing every corpus sentence) that is necessary to answer a query for any input sentence. In the presence of multiple queries, it would be much better to construct a table of the corpus sentences and their tokens only once, and then use this table to answer each input query. We can simply reorder and fold the above program clause to create and use that corpus_word/2 relation as follows:

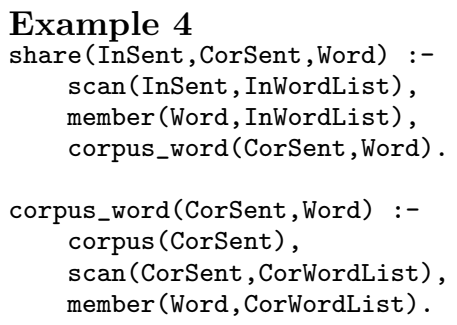

This separates out the relation we want to generate and reuse, but notice that variant tabling does not solve our redundant computation problem. The predicate corpus_word/2 will be called with its second argument, Word, bound. So for each different word in the input sentence, corpus_word/2 will be entered, thus re-scanning every corpus sentence for each new input word. This is even worse than the previous unfolded program.

However, if we can ensure that the corpus_word/2 definition is evaluated bottom-up, generating a complete table that is indexed on its second argument and that table is used for every call from share/3, then the redundant computation will be eliminated.

So this is an example of a situation in which we want to build (i.e., initialize) a table containing more answers than we immediately need: we want answers computed for all corpus words, even though we initially need only those for a single word. 
In procedural terms, we understand the bottom-up evaluation of corpus_word/2 as building an intermediate data structure to more efficiently evaluate various subqueries that will be needed later. This is a common requirement in algorithm development. Experienced Prolog programmers normally implement such requirements non-declaratively by initially computing the necessary tuples and adding them to a dynamic predicate using the Prolog built-in assert/1. But with bottom-up evaluation, such programs can be purely declarative and yet have the equivalent computational properties.

\section{Specifying Bottom-Up Evaluation in XSB}

The question now is how to indicate to the evaluation system that the first call to corpus_word/2 is to cause it to be evaluated bottom-up to fill the table that is to be indexed on its second argument, and how the evaluation system will efficiently carry out that evaluation.

XSB uses Subsumptive Tabling With Abstraction (STWA) to carry out the bottom-up evaluation. Normally tables in XSB are variant tables, i.e. an entry in a variant table is used to satisfy a subsequent goal if that goal is a variant of the goal that generated the table entry, i.e., equal up to change of variable. In subsumptive tabling (Johnson et al. 1999; Ramakrishnan et al. 1999; Johnson 2000; Johnson 2002; Cruz and Rocha 2011), a table entry is used to satisfy a subsequent goal if the subsequent goal is subsumed by the generating goal, i.e., is an instance of the generating goal. Note that in such a case the answers to the subsumed goal will (eventually) be in the table of answers for the previously encountered subsuming goal. ${ }^{1}$ The programmer may specify, using a table_index/2 directive, that when a subsumptively tabled goal is called, the calling goal should be abstracted before it is actually invoked, so that a table for the more general call is constructed, and then only the answers unifying with the original unabstracted goal are returned to the initial call. In this case, since a general table is constructed, a later subgoal that is subsumed by that more general goal will use this general table to satisfy the later subgoal and not make the specific subgoal call to do clause resolution.

The table_index/2 directive was recently added to XSB to support the creation of subsumptive tables with multiple arbitrary indexes (Warren et al. 2007). An index on a single argument of the table is specified by just giving an integer that indicates the position in the tabled predicate of the argument to be indexed. Joint indexes, i.e. indexes on multiple arguments, are specified by terms made by separating the multiple position integers by the symbol + . Multiple different indexes are provided in a list. (These index specifications are the same as those used for dynamic predicates in XSB.) For example, say we want four indexes on a subsumptive table for $\mathrm{p} / 4$, so that a call to $\mathrm{p} / 4$ (after its table is built) first checks if the call has arguments 1 and 2 bound and if so uses a joint index on those arguments; and if not, then checks if it has just argument 1 bound and if so uses an index on that; and if not then checks if arguments 2, 3 and 4 are bound and if so uses a joint index on those positions; and if not then checks if argument 4 is

1 Not all predicates defined in Prolog can be correctly tabled. For example, variant tabled predicates should not use built-ins that change the global state, such as assert/1 or retract/1; subsumptively tabled predicates, in addition, should not use built-ins that depend on the instantiation state of variables, such as var/1 and many uses of the cut (!) operation. 
bound and if so uses an index on that position; and if not, then throws an error (because that would be an unindexed call and we should be notified). So this would require the directive:

:- table_index $(\mathrm{p} / 4,[1+2,1,2+3+4,4])$.

The table_index/2 directive not only specifies the indexes on the resulting subsumptive table but also what abstraction should be done to the initial call to the subsumptively tabled predicate. The assumption is that every index will have some calls that use it. Since we want to compute the tuples for only one abstracted call to the tabled predicate, every position that might be unbound on some particular call must be abstracted in the initial subsumptive call, so that the one table will contain all answers for a call not bound on that position. This implies that the arguments that should be bound in the abstracted call to the subsumptively tabled predicate are those that appear in every specified index; all others must be variables. For the p/4 example, there are no such arguments, so the first call will be fully abstracted, i.e., all arguments will be variables. In this case we call it "subsumptive tabling with full abstraction".

There are situations where we want the initial call to a subsumptively tabled predicate to be abstracted to the fully open call, even if there are positions appearing in all index specifications. For example, we may need only one index but want full abstraction, as in our example for corpus_word/2. In such a case, we must use 0 as an index specifier, meaning "no index", and it is placed last.

Thus for the corpus_word/2 subsumptive table, we want:

:- table_index(corpus_word/2, $[2,0]$ ).

which declares that the predicate corpus_word/2 should be computed bottom-up. We want an index on the second argument and the first call should be abstracted to the call with all variables. Since every corpus_word/2 subgoal is subsumed by this most general goal, it will never be called after the first time, but the table that is constructed at the first call will be used to satisfy all those subsequent calls. The first call to corpus_word/2 will build (or initialize) the table (or data structure), and from then on every call will directly access that table; and in our case use the index for constant time lookup.

The directive table_index/2 is implemented in XSB by a program transformation, which we briefly describe by example. The following code (slightly modified for clarity) is generated by the compiler for the $\mathrm{p} / 4$ example above,

:- table_index $(\mathrm{p} / 4,[1+2,1,2+3+4,4])$ :

:- table p1234/4, p4231/4 as subsumptive.

$\mathrm{p}(\mathrm{A}, \mathrm{B}, \mathrm{C}, \mathrm{D})$ :-

nonvar $(A) \rightarrow p 1234(A, B, C, D)$

; nonvar(D) $\rightarrow$ p4231(D, B, C,A)

; table_error('Illegal Mode in call to $\mathrm{p} / 4^{\prime}$ ').

p1234 (A, B, C, D) :-

$\operatorname{var}(A), \operatorname{var}(B), \operatorname{var}(C), \operatorname{var}(D)->$ P_base $(A, B, C, D)$

; $\mathrm{p} 1234(\mathrm{E}, \mathrm{F}, \mathrm{G}, \mathrm{H}), \mathrm{E}=\mathrm{A}, \mathrm{F}=\mathrm{B}, \mathrm{G}=\mathrm{C}, \mathrm{H}=\mathrm{D}$.

p4231 (A, B, C, D) :-

$\operatorname{var}(\mathrm{D}), \operatorname{var}(\mathrm{B}), \operatorname{var}(\mathrm{C}), \operatorname{var}(\mathrm{A}) \rightarrow \mathrm{p} 1234(\mathrm{D}, \mathrm{B}, \mathrm{C}, \mathrm{A})$

; $\mathrm{p} 4231(\mathrm{E}, \mathrm{F}, \mathrm{G}, \mathrm{H}), \mathrm{E}=\mathrm{A}, \mathrm{F}=\mathrm{B}, \mathrm{G}=\mathrm{C}, \mathrm{H}=\mathrm{D}$.

p_base $(A, B, C, D):-\ldots$ original $p$ rules ...

p1234/4 and p4231/4 are versions of p/4 with its arguments permuted. Subsumptive tables implement trie indexing, thus providing indexing on any initial sequence of arguments. Here two tables are needed: p1234/4 for indexes 1 and $1+2$, and p4231/4 
for indexes 4 and $2+3+4$. (The complier determines the minimum number of tables required to cover all the declared indexes.) The first clause tests the calling mode to select which tabled permutation to call. Each permutation predicate will normally be entered twice, once with some arguments bound, which will result in a (second) call to itself with all arguments unbound. From then on every other call will be subsumed by that most general call and so its answers will be served from that generated table. Notice that p4231/4 calls p1234/4 and not p_base/4 and so will be filled by getting its answers from the table of $\mathrm{p} 1234 / 4$. Thus the original clauses that define $\mathrm{p} / 4$ (now in $\mathrm{p}$ _base/4) will be called only once.

\section{Intermixing Top-down and Bottom-up evaluation}

It is possible to combine top-down filtering with bottom-up evaluation.

\section{Example 5}

Say our corpus of sentences in Example 3 is partitioned to indicate those from a single book. We would have another argument to corpus/1 now:

corpus (BookISBN, Corent). And top-level predicate:

share (InSent, BookISBN, CorSent), and sub-predicate:

corpus_word (BookISBN, CorSent, Word), defined in terms of corpus/2.

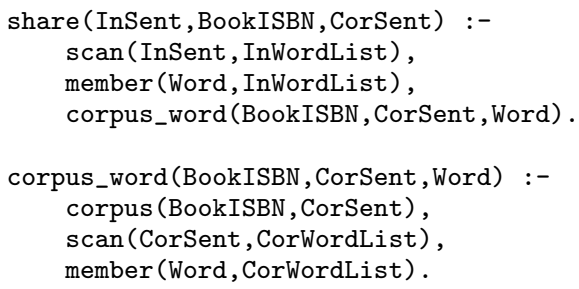

We assume that corpus/2 is indexed on its first field, BookISBN, and require that BookISBN be bound on a call to share/3. Then we would invoke partial bottom-up evaluation with the declaration:

:- table_index(corpus_word/3, $[1+3,1]$ ).

This will abstract a call to corpus_word/3 to corpus_word (+,-,-), where + indicates a bound argument and - a variable argument. Positions that appear in all indexes in the table_index declaration, here 1, will not be abstracted; all others will be. Now the sentences of each book will be processed bottom-up independently. When a new book is queried for the first time, the table for words in sentences for that book will be computed bottom-up and stored in the table. Subsequent queries to the same book will directly use the constructed table. So this is an example in which demand-driven computation (here with respect to BookISBN) can be combined with data-driven computation (here for the set of corresponding < CorSent, Word $>$ pairs) to evaluate a query (here to share/3.)

That a data-driven computation can use a demand-driven subquery has already been seen in the original Example 3. While we haven't shown the code for the scan/2 predicate, it is naturally defined using demand-driven computation. E.g., a DCG defining the scanner would use top-down evaluation of a subquery. So the original example shows how the bottom-up evaluation of corpus_word $(-,-)$ uses the top-down evaluation of $\operatorname{scan}(+,-)$. 


\section{Procedural Interpretation of Bottom-up Evaluation}

To understand top-down evaluation of rules, one intuitively thinks of subgoal solving as nondeterministic procedure invocation, with backtracking to explore alternate computation paths. To understand top-down evaluation with tabling, one thinks of procedure invocation but with short-circuiting for previously encountered procedures (with the same parameters). To understand bottom-up evaluation as provided by Subsumptive Tabling With Abstraction (STWA) through the table_index/2 directive, one thinks of indexed table construction (or initialization) from which future retrieval will be done.

A bottom-up computation is appropriate when there is a predicate that is efficiently computable in one mode and yet it will be needed multiple times in another mode. For example, corpus_word (+.-) of Example 3 can be efficiently evaluated in that direction, i.e., given a sentence find the words in it. But the application requires multiple calls of the mode corpus_word $(-,+)$, i.e., given a word find the sentences that contain it. In this situation, bottom-up evaluation is called for.

The most basic example of this phenomenon is indexing itself. For example, if one wants to find the value associated with a particular key in an unordered sequence of keyvalue pairs, one must search the pairs sequentially, which on average takes time linear in the number of pairs. With the same time complexity to search for one item, one can (with perfect hashing) build a hash table which allows all such key lookups to be done in constant time.

\section{STWA for File Input}

Part of the attractiveness of logic programming is that it treats programs (usually mostly rules) and data (usually facts) the same, but in most large systems there is an important pragmatic difference between them: Programs are rules (and facts) that are known statically at compile time; data are facts (and maybe rules) that are not known until runtime when they are retrieved from a file. Facts are normally accessed through a filename passed to an executing program, where the records of the file are initially read and asserted into a predicate in memory, which is used by the program rules. It is not clear how this could be made fully declarative. But consider the following use of STWA:

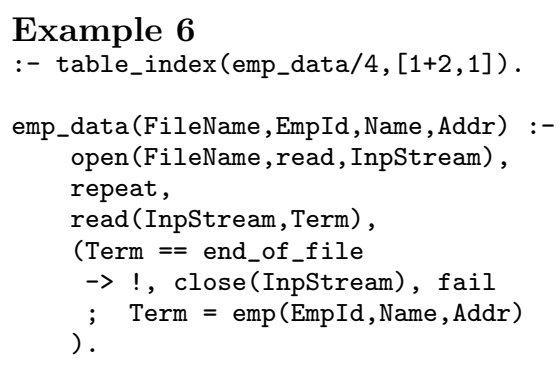

This definition uses low-level procedural Prolog operations to read the records of a file containing emp $/ 3$ facts and return them as answers to a call to emp_data/4, where the first argument of the call is the name of the file to read. Because of the table_index/2 directive, the first call will be abstracted to emp_data $(+,-,-,-)$. (Recall that arguments in the intersection of all the indexed arguments, here argument 1, must be bound in any call, and will not be abstracted for the initial table-filling call.) So with this 
definition, a programmer can get access to the tuples in any employee file (one that contains emp/3 facts) by invoking a simple subgoal, with the first argument providing the name of the file. There is no need for the programmer to make an explicit call (e.g., Prolog's ensure_loaded/1) to initialize the emp_data/4 predicate using Prolog's nonlogical assert/1. The first call will automatically read the file in, building an index on the first and on the first and second arguments. Since XSB uses tries to represent tables (Ramakrishnan et al. 1999), this is here just a single trie, and the Filename is stored only once in the table, so the overhead of using the 4-ary predicate is essentially zero.

Of course a programmer could read different sets of employee facts from different files simply by invoking the emp_data goal with different file names.

Generalizing this approach, we can make the entire file system a part of the dataspace of a Prolog program. Definitions such as in Example 6 simply map (portions of) the file system into Prolog fact-defined predicates. We can make this easier for the programmer by providing a single "file-system" predicate that views the entire file system as a single relation. For example, consider a system predicate (Warren et al. 2007): data_records (+FileName, +FileFormat, ?RecordTerm), which, given a filename and a file format, reads the file, parses each line/record to a Prolog term as specified by FileFormat, and returns each such term nondeterministically in RecordTerm.

Now a Prolog programmer can use table_index/2 and more easily define emp_data/4 as:

:- table_index (emp_data/4, $[1+2,1])$.

emp_data (FileName, EmpId, Name, Addr) :-

data_records (FileName, read, emp (EmpId, Name, Addr)).

to cause the emp_data/4 predicate to be loaded from the named datafile on its first call. With this framework, the Prolog programmer has declarative access to all data in the file system, without having to worry explicitly about how and when to load it into memory.

\section{Does Subsumptive Tabling with Abstraction (STWA) Really Do Bottom-Up Evaluation?}

Subsumptive tabling with abstraction has the properties of bottom-up evaluation, described above, of generating a full table once and then using the resulting table for subsequent queries. But does it actually build that table using the well-understood bottom-up, forward-chaining, data-directed algorithm, as described in Section 2? The short answer is yes (mostly), which we now demonstrate.

\section{Example 7}

Consider how XSB would compute the transitive closure of Example 2 under STWA. The program is:

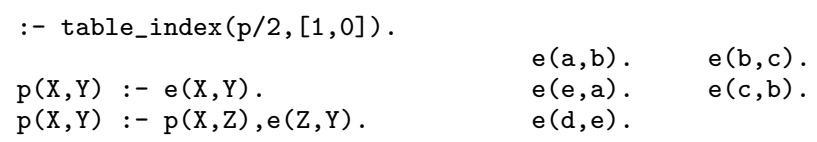

Instrumented to produce output showing the engine's progress (see ??), we get (with annotations added for clarity): 


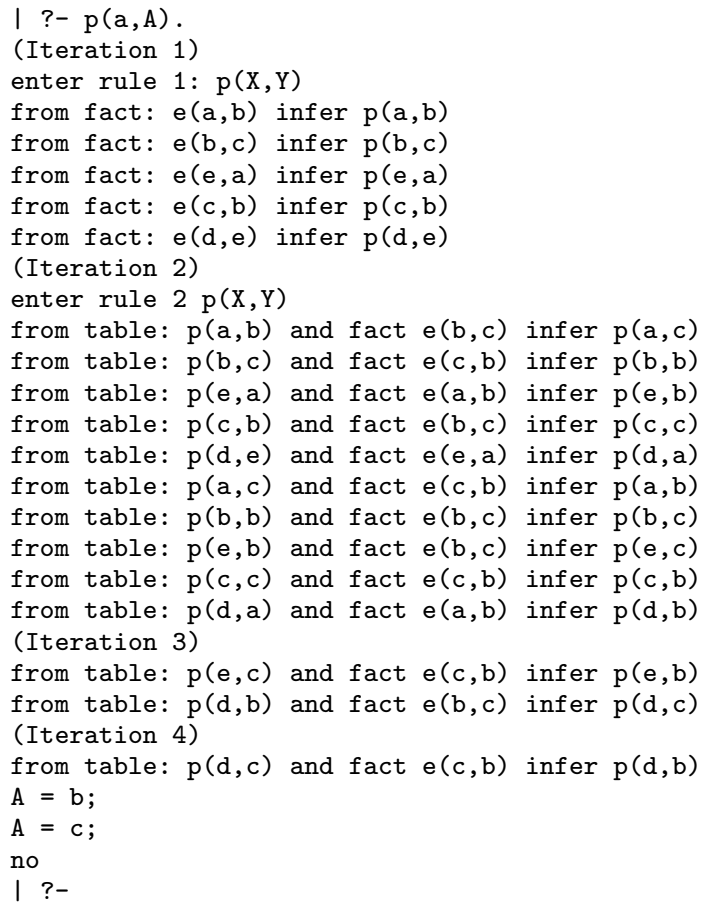

This log closely follows that of Example 2: the "enter rule" lines show that each rule (and thus the predicate) is entered only once. From then on everything is returned through the table for $\mathrm{p}(\mathrm{X}, \mathrm{Y})$. The only differences are the repeated answers in Iteration 2 , where duplicate answers are computed but not added to the table. (Were we more detailed in our description of Example 2, these would have appeared there, too.)

Intuitively, what happens here is that the initial call to $\mathrm{p}(\mathrm{a}, \mathrm{A})$ is fully abstracted to $\mathrm{p}(\mathrm{X}, \mathrm{Y})$ and that call is made. The first rule returns all its answers to the table, which we have labeled "Iteration 1". Then the second rule is entered and the open call to $\mathrm{p}(\mathrm{X}, \mathrm{Z})$ (the first subgoal of that second rule) is made, found to have previously been made, and suspended, to return answers from the table. The rest of the log, of iterations 2 through 4 , consists of answers from the table being returned to that call, joined with facts e/2 and the resulting answers added (if new) to the table. So computation across the bodies of rules may be suspended to wait for new answers to show up in subsumptive tables. This, in effect, uses XSB's scheduling queues to get the equivalent of semi-naive bottom up computation using pointers. This computation directly mirrors the bottom-up evaluation of the query $\mathrm{p}(\mathrm{X}, \mathrm{Y})$.

\subsection{Multiple Machine Model of Tabled Horn Clause Evaluation}

We describe a model for STWFA evaluation as a set of (virtual) machines. Given a Horn clause program and a goal, each machine carries out the evaluation of the goal in the program along a different deterministic path. Without tabling there would be a machine corresponding to each root-to-leaf path of the SLD tree for the query. With subsumptive tabling with full abstraction, the model is as described next. 
A global table of subgoals is maintained. Associated with each subgoal is a set of answers corresponding to proven instances of the subgoal. This table is maintained and used by all the executing machines. It is monotonic in that goals and answers are only added to the table and never deleted.

Computation starts with a single machine that is given the initial goal to evaluate. When a machine encounters a goal, it looks to see if the corresponding most-general goal is in the global table. If it is not in the table, a) it adds it (with an empty list of associated answers), b) for each clause for that most-general goal, it forks off a duplicate of itself to process it, and c) remembering the goal it encountered, it suspends on the table entry it just added (to later process answers that are associated with that most-general goal). If the most-general goal already is in the table, the machine (remembering its encountered goal) suspends on that table entry.

When a machine encounters a failure, it simply disappears. When a machine returns an answer to a goal (i.e., completes execution to the end of a clause for that goal), it adds the computed goal instance to the end of the list of answers associated with that goal in the table, and then disappears.

Whenever there is a new answer for a table entry for a goal, each machine that is suspended on that entry, whose associated goal unifies with that new answer, forks off a copy of itself. This new machine uses that answer to update its state and then continues executing. The suspended machine remains suspended having marked in the table that that answer has been returned.

This set of machines is normally simulated by a sequential emulator, which includes a scheduler that determines which of the next possible machine operations will be performed. At any point in the execution, there may be a machine ready to a) evaluate the next goal of some clause (or return an answer if there are no more goals for that clause) or b) fork a machine that is suspended on a table entry to process a new (unifying) answer. For specificity and simplicity, we will assume that answers associated with a table entry are maintained in the order they are generated, and suspended machines return them in that same order.

\section{Theorem 1}

Assume $\mathrm{H}$ is a Horn program and $\mathrm{P}$ a predicate in $\mathrm{H}$ such that all predicates in $\mathrm{H}$ are reachable from $\mathrm{P}$ in the predicate call graph of $\mathrm{H}$. (I.e., the goal predicate potentially depends on all predicates.) Assume that for every rule body in $\mathrm{H}$, there is some instance of it that is true in the least model of $\mathrm{H}$. Then evaluation of predicate $\mathrm{P}$ in $\mathrm{H}$ under Subsumptive Tabling With Full Abstraction (STWFA) is equivalent to a bottom-up computation of the least model of $\mathrm{H}$.

\section{Proof}

(Sketch) We formulate a bottom-up Horn clause evaluator and then compare it to the multiple machine model of STWFA described above.

Consider the following formulation of a bottom-up Horn clause evaluator. For each clause, there is a process to generate facts for instances of its head atom. There is a global set of proven facts that is initialized to empty. The rule processes run as follows: Each rule process looks to find an instance of its clause such that all its body atoms appear in the global fact set. For each such clause instance, it adds the head atom to the 
global fact set, if it is not already a member. The processes continue to run to a fixpoint, i.e., until no process can add any new fact to the global set.

By synchronizing and scheduling the rule processes so that they iteratively generate a new set of facts to be added to the fact set by using facts only from earlier iterations, one obtains the well-known iterative bottom-up evaluation algorithm.

However, any order of execution of the rule processes is possible, and constitutes an evaluation method appropriately called bottom-up.

In STWFA, a machine that is generated for a rule when its most-general subgoal is first entered corresponds to a rule process in the bottom-up algorithm. The execution of such a machine (and its descendants), which suspend and fork off new machines to process new answers, corresponds to the bottom-up rule process for the clause that finds rule instances whose body atoms are in the global fact set. Finally, a machine returning a new answer to a predicate corresponds to a rule process adding a new fact to the global fact set.

The difference between the two algorithms is how the rule processes/machines get set up in the first place. For the bottom-up algorithm, the rule processes are set up at the same time during an initialization phase. With STWFA, the corresponding machines for the rules of a particular predicate are generated at the first call to a goal for that predicate. STWFA will generate relations only for predicates on which the goal predicate depends, since it will not initialize rule processor machines for other predicates that are never called.

The theorem requires that the goal depends on all predicates in the program, and that every clause has a true instance in the least model of the program. This will guarantee that STWFA will eventually call every predicate and so all rule-processing machines will be initialized.

We can fix a scheduling order for STWFA, and then find an equivalent scheduling order for bottom-up evaluation with rule processes.

\section{Bottom-up Proofs for Propositional Horn Clauses in Linear Time}

For a deeper understanding of XSB's STWA and bottom-up evaluation, we consider the well-known three-line meta-interpreter, applied to propositional programs.

\section{Example 8}

Consider the (folded variation of the) classic meta-interpreter of Prolog programs:

interp (true).

$\operatorname{interp}((A, B)):-\operatorname{interp}(A)$, interp(B).

$\operatorname{interp}(G):-\operatorname{interpAtom}(G)$.

interpAtom(G) :- (G<- Body), interp(Body).

It is well-known that if we add $<-/ 2$ facts to define a (propositional) Horn clause program and evaluate interp(Goal) for some Goal in Prolog, this will carry out the top-down Prolog evaluation of Goal with respect to the program. Similarly, if we add the declaration:

:- table interpAtom/1.

this will carry out top-down evaluation with (variant) tabling. 
Now consider instead adding the declaration:

:- table_index(interpAtom/1, $[1,0]$ ).

and posing the query: interpAtom(Prop), with Prop a variable. Recall that this declaration causes XSB to use subsumptive tabling. There is no abstraction necessary for this query since it is already fully abstract, so all subsequent calls to interpAtom/1 will be served from the table constructed by that initial call. The claim is that this will cause XSB to evaluate the propositional program in $(<-) / 2$ using a bottom-up algorithm. To see this, consider how this query will be processed. Note that since there is only the one call to interpAtom(_), there can be no propagation of demand through (partially) instantiated arguments. The initial goal is interpAtom(_), the open call. So this initial call, being subsumptively tabled, will add the call to the table and invoke the predicate code to do clause resolution. Now every other call to interpAtom(P) for any particular proposition $\mathrm{P}$ (here only the one in the third clause of interp/1) will not invoke the code for interpAtom/1, but will return answers that have previously been added to the table and will suspend waiting for a new answer to show up in the table to then be returned. The first answers returned cannot require a recursive call to interpAtom/1, so they can come from only the first clause of interp/1, and those will be the facts of the program in $(<-) / 2$. So the first answers to show up in the table for interpAtom/1 will be the program facts, just as in bottom-up evaluation. Then those facts can be returned to the recursive call to interpAtom/1 (in the third clause of interp/1.) So rules all of whose body atoms are facts will have their recursive calls to interpAtom/1 satisfied (since they will be in the interpAtom(_) table on which they are waiting), and will succeed returning the propositions in their heads, to be added to the table for interpAtom(_). And so forth.

We can see the order that XSB generates the propositional answers to our program above by executing the above meta-interpreter (with logging operations, as shown in ??):

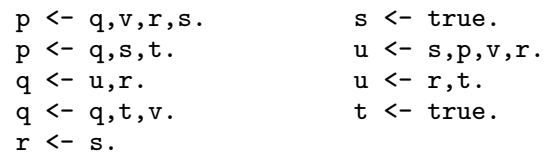

where we get (lightly edited):

$\begin{array}{ll}\text { 1 } ?-\text { interpAtom }(\mathrm{p}) \text {. } \\ \text { 1. Var demanded } \\ \text { 2. } \mathrm{p}<-\mathrm{q}, \mathrm{v}, \mathrm{r}, \mathrm{s} & \text { initial clause } \\ \text { 3. } \mathrm{p}<-\mathrm{q}, \mathrm{s}, \mathrm{t} & \text { initial clause } \\ \text { 4. } \mathrm{q}<-\mathrm{u}, \mathrm{r} & \text { initial clause } \\ \text { 5. } \mathrm{q}<-\mathrm{q}, \mathrm{t}, \mathrm{v} & \text { initial clause } \\ \text { 6. } \mathrm{r}<-\mathrm{s} & \text { initial clause } \\ \text { 7. } \mathrm{s}<- \text { true } & \text { initial clause } \\ \text { 8. } \mathrm{s} & \text { from true and } \mathrm{s}<- \text { true } \\ \text { 9. } \mathrm{u}<-\mathrm{s}, \mathrm{p}, \mathrm{v}, \mathrm{r} & \text { initial clause } \\ \text { 10. } \mathrm{u}<-\mathrm{p}, \mathrm{v}, \mathrm{r} & \text { from } \mathrm{s} \text { and } \mathrm{u}<-\mathrm{s}, \mathrm{p}, \mathrm{v}, \mathrm{r} \\ \text { 11. } \mathrm{u}<-\mathrm{r}, \mathrm{t} & \text { initial clause } \\ \text { 12. } \mathrm{t}<-\mathrm{true} & \text { initial clause } \\ \text { 13. } \mathrm{t} & \text { from true and } \mathrm{t}<-\mathrm{true} \\ \text { 14. } \mathrm{r}<\mathrm{from} \mathrm{s} \text { and } \mathrm{r}<-\mathrm{s} \\ \text { 15. } \mathrm{u}<-\mathrm{t} & \text { from } \mathrm{r} \text { and } \mathrm{u}<-\mathrm{r}, \mathrm{t} \\ \text { 16. } \mathrm{u} & \text { from } \mathrm{t} \text { and } \mathrm{u}<-\mathrm{t} \\ \text { 17. } \mathrm{q}<-\mathrm{r} & \text { from } \mathrm{u} \text { and } \mathrm{q}<-\mathrm{u}, \mathrm{r} \\ \text { 18. } \mathrm{q} & \text { from } \mathrm{r} \text { and } \mathrm{q}<-\mathrm{r} \\ \text { 19. } \mathrm{q}<-\mathrm{t}, \mathrm{v} & \text { from } \mathrm{q} \text { and } \mathrm{q}<-\mathrm{q}, \mathrm{t}, \mathrm{v} \\ \text { 20. } \mathrm{q}<-\mathrm{v} & \text { from } \mathrm{t} \text { and } \mathrm{q}<-\mathrm{t}, \mathrm{v}\end{array}$




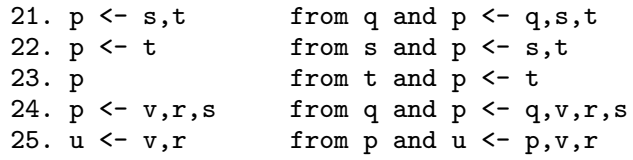

Each line (from 2 on) shows a derived clause that is either an initial program clause, or derived from a previous clause by removing the first proposition in its body if it is proved (or is the constant 'true'). A proposition is proved if it is the head of a derived clause with an empty body. Each step is the result of a unit resolution, whose participating clauses are indicated on the line. Unit resolution generates a form of bottom-up propositional reasoning for Horn clauses.

Performing iterative bottom-up evaluation of this program, we get:

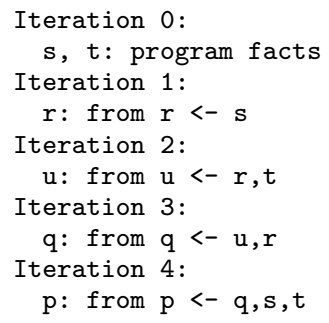

And we see these propositions showing up in exactly this order in the XSB STWA log above.

\section{Performance of XSB STWA for Bottom-Up Evaluation}

In this section we show XSB's performance on bottom-up evaluation of a propositional program using a meta-interpreter. The system used is XSB Version 3.8.0 on a Mac Pro with an i7-4870HQ CPU running at 2.5-GHz with 16 GB of RAM and the 64-bit Windows 10 Operating System. The program we use is a "triangular" program, of the form:

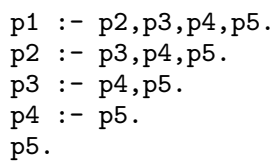

This program is an example with 5 rules (and facts) and 15 proposition occurrences. The meta-interpreter is:

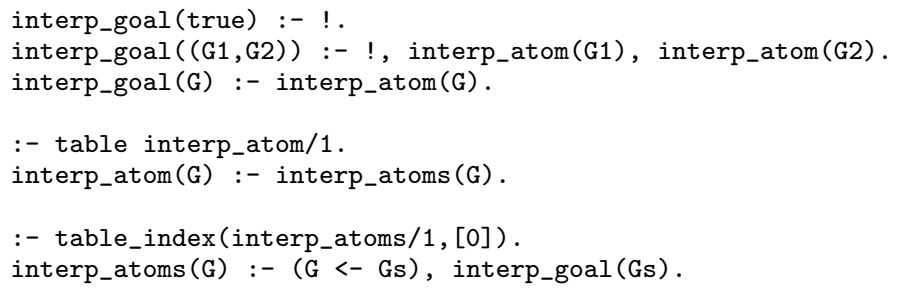

Notice that we use both a variant table (on interp_atom/1) and a subsumptive table (on the equivalent interp_atoms/1.) This is to improve efficiency, since lookup and 


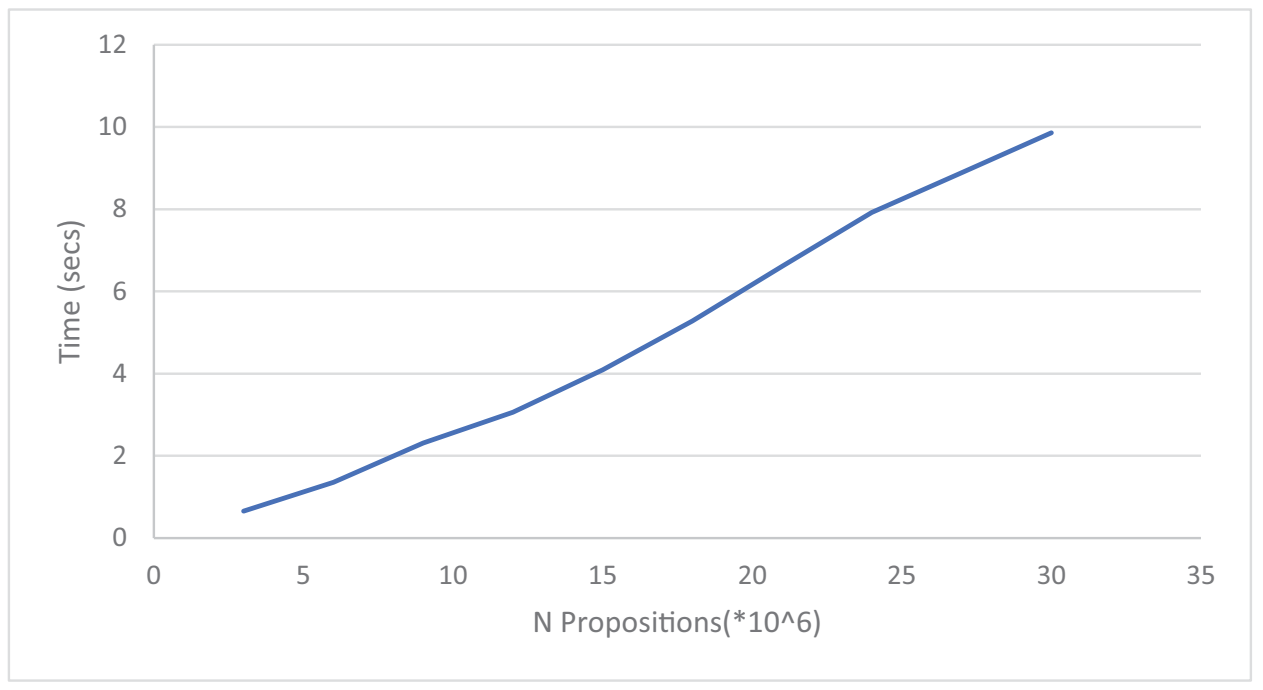

Fig. 1. Times for Bottom-up Evaluation of Triangular Programs

retrieval from a variant table in XSB is significantly faster than for a subsumptive table. ${ }^{2}$ And in these triangular programs there are many lookups of the same variant call; each proposition is looked up once per occurrence in the body of a rule, so on average there are approximately NumRules/2 lookups per proposition, so lookups completely dominate the computation time.

Figure 1 shows the cpu time for bottom-up evaluation of various-sized triangular programs. $N$ corresponds to the number $\left(* 10^{6}\right)$ of proposition occurrences in the program, or more precisely, the number in the largest triangular program that has $N * 10^{6}$ or fewer proposition occurrences.

Times are just for the meta-interpretation of the triangular programs; the time for generating the $(<-) / 2$ facts for the programs is not included. The graph is essentially a straight line, showing the linearity of the algorithm. The propositional Horn clause satisfaction problem is known to be linear in the number of proposition occurrences (Dowling and Gallier 1984), and this meta-interpreter is indeed linear on all propositional logic programs.

Triangular programs have no loops so pure top-down evaluation without tabling will terminate, but will be very slow due to many redundant computations. For example to evaluate without tabling the single query, p1, to a triangular program with 325 proposition occurrences takes c. 4.1 seconds, and with 465 occurrences c. 126 seconds. This contrasts with the 15,000,000 occurrences in the program that is computed by tabled bottom-up evaluation in 4.1 seconds (as shown in the figure).

2 Without the additional variant table, the evaluation is about 25 times slower, showing significant room for improvement in XSB's implementation of subsumptive table goal lookup. Evaluation of this program with only variant tabling is about twice as fast; not surprising since that evaluation is also linear, it doesn't duplicate tables, and XSB is optimized for variant tables. 


\section{Conclusion}

In this paper we have described the integration of bottom-up evaluation with top-down evaluation in tabled Prolog by means of Subsumptive Tabling With Abstraction (STWA). We provided a procedural interpretation for bottom-up evaluation, in the context of Prolog programming, as efficient indexed data structure initialization. This allows many formerly non-declarative Prolog programs that contain assert/1 to be naturally expressed as declarative programs with no sacrifice in efficiency.

We have shown how XSB provides a natural model for evaluating Horn programs, based on nondeterministic procedural interpretation using subsumptive tabling with abstraction, that incorporates both top-down and bottom-up computation in a single uniform framework. And this model provides linear evaluation of all queries to proposition Horn programs for all intermixings of demand and data driven computation.

\section{References}

Aref, M., ten Cate, B., Green, T. J., Kimelfeld, B., Olteanu, D., Pasalic, E., Veldhuizen, T. L., And Washburn, G. 2015. Design and implementation of the logicblox system. In Proceedings of the 2015 ACM SIGMOD International Conference on Management of Data. SIGMOD '15. ACM, New York, NY, USA, 1371-1382.

Arni, F., Ong, K., Tsur, S., Wang, H., and Zaniolo, C. 2003. The deductive database system L++. Theory and Practice of Logic Programming 3, 1 (Jan.), 61-94.

BRY, F. 1990. Query evaluation in deductive databases: Bottom-up and top-down reconciled. Data Knowledge Engineering 5, 289-312.

Cruz, F. And Rocha, R. 2011. Efficient instance retrieval of subgoals for subsumptive tabled evaluation of logic programs. CoRR abs/1107.5556.

Dowling, W. F. And Gallier, J. H. 1984. Linear-time algorithms for testing the satisfiability of propositional horn formulae. J. Log. Program. 1, 267-284.

Hermenegildo, M. V., Bueno, F., Carro, M., López-García, P., Mera, E., Morales, J. F., And Puebla, G. 2012. An overview of Ciao and its design philosophy. TPLP 12, 1-2, 219-252.

Johnson, E. 2000. Interfacing a tabled-wam engine to a tabling subsystem supporting both variant and subsumption checks. In 2nd Workshop on Tabulation in Parsing and Deduction. Vigo, Spain.

Johnson, E. 2002. A system supporting both variant- and subsumption-based tabled evaluations of logic programs. Ph.D. thesis, Stony Brook University, Stony Brook, NY, 117944400 .

Johnson, E., Ramakrishnan, C. R., Ramakrishnan, I. V., and RaO, P. 1999. A space efficient engine for subsumption-based tabled evaluation of logic programs. In Functional and Logic Programming: 4th Fuji International Symposium, FLOPS'99 Tsukuba, Japan, November 11-13, 1999 Proceedings, A. Middeldorp and T. Sato, Eds. Springer Berlin Heidelberg, Berlin, Heidelberg, 284-299.

Kowalski, R. 1986. Logic for Problem-solving. North-Holland Publishing Co., Amsterdam, The Netherlands, The Netherlands.

Lloyd, J. W. 1993. Foundations of Logic Programming, 2nd ed. Springer-Verlag New York, Inc., Secaucus, NJ, USA.

Ramakrishnan, I. V., Rao, P., Sagonas, K., Swift, T., and Warren, D. S. 1999. Efficient access mechanisms for tabled logic programs. Journal of Logic Programming 38, 1 (Jan), $31-54$. 
Ramakrishnan, R., Srivastava, D., Sudarshan, S., and Seshadri, P. 1994. The CORAL deductive system. VLDB J. 3, 2, 161-210.

Santos Costa, V., Rocha, R., And Damas, L. 2012. The YAP prolog system. TPLP 12, 1-2, $5-34$.

Swift, T. And Warren, D. S. 2012. XSB: Extending Prolog with tabled logic programming. The Theory and Practice of Logic Programming 12, 1-2 (January), 157-187. (http://journals.cambridge.org/repo_A84WadE7).

Tekle, K. T. And Liu, Y. A. 2011. More efficient datalog queries: Subsumptive tabling beats magic sets. In Proceedings of the 2011 ACM SIGMOD International Conference on Management of Data. SIGMOD '11. ACM, New York, NY, USA, 661-672.

Toman, D. 1995. Top-down beats bottom-up for constraint extensions of datalog. In In Proc. Intl. Logic Programming Symposium. MIT Press, 98-114.

Ullman, J. D. 1989. Bottom-up beats top-down for datalog. In Proceedings of the Eighth ACM SIGACT-SIGMOD-SIGART Symposium on Principles of Database Systems. PODS '89. ACM, New York, NY, USA, 140-149.

WArren, D. S. 1992. Memoing for logic programs. CACM 35, 3 (Mar), 93-111.

Warren, D. S., Swift, T., And SAgonas, K. F. 2007. The XSB programmer's manual. Tech. rep., Department of Computer Science, State University of New York at Stony Brook, Stony Brook, New York, 11794-4400. Mar. The XSB System, current version 3.8.0, Nov 2017, is available from xsb.sourceforge.net, and the system and manual are continually updated.

Weilemaker, J., Frehwirth, T., And De Koninck, L. 2017. SWI-Prolog reference manual. Tech. rep. Tabling library available at http://www.swi-prolog.org/pldoc/man?section= tabling.

Zhou, N. 2012. The language features and architecture of B-Prolog. TPLP 12, 1-2, 189-218. 